\title{
Comparative Study on the Analysis Methods for the Seismic Resistance of Bridge Structure
}

\author{
Danling Wang \\ Hehai College, Chongqing Jiaotong University, Chongqing, China 400041
}

\begin{abstract}
As a kind of destructive natural disasters, earthquake can cause serious damage to the bridges of lifeline projects, which will bring great difficulties to the rescue and relief work. The bridge structure is complex, and people pay increasingly more attention to the research on its seismic resistance. It is very important for designers and researchers to adopt an appropriate analysis method in seismic resistance analysis. This paper briefly summarizes the hazards caused by earthquakes to long-span bridges, and introduces the calculation principles of response spectrum method and time history analysis in detail. Through the comparative study of the two commonly used seismic resistance analysis methods for bridges, it summarizes the advantages and disadvantages and the application scope of each method, providing reference for selecting suitable design methods for seismic resistance design. Lastly, it describes the future research trends of response spectrum method and time history analysis.
\end{abstract}

Keywords: Seismic resistance of bridge; Response spectrum method; Time history analysis method.

\section{Introduction}

In recent years, devastating earthquakes occur frequently around the world, which seriously threatens the lives and property of human beings. With the rapid development of science and technology and transportation, bridge engineering is an important public transportation hub of modern society, so the damage to it would cause great difficulties for post-earthquake rescue and post-disaster reconstruction and seriously affect the operation of social production, resulting in huge economic loss.

In order to meet the needs of social and economic development, bridge construction in China in recent decades develops rapidly, and the correct seismic resistance design of bridge projects is a major event concerning the national economy and people's livelihood. Seismic resistance of bridges is an important part of the design process, ensuring that bridge engineering has strong seismic resistance performance and mitigates damage caused by earthquakes, which has become an issue that academics and engineering circles attach great importance to[1].

At present, analysis methods for bridge structures' seismic resistance mainly include response spectrum method and time history analysis method. This paper first introduces the calculation principles of the above two analytical methods, conducts comparative study on the two analytical methods, and summarizes the advantages and disadvantages and the application scope of each method, to provide reference for the selection of analysis methods of bridges' seismic resistance design.

\section{Calculation principles}

\subsection{Response spectrum method}

In 1941, Bito proposed the concept of response spectrum, and gave the first elastic response spectrum for the first time. In the late 1940s and early 1950s, Huosner et al. proposed a theory of structural seismic resistance analysis based on response spectrum on the basis of calculating a large number of response spectra. The establishing bases of the response spectrum analysis model are effective stiffness characteristics and equivalent viscous damping ratio assumption. The assumed conditions are: (1) the cracking or effective stiffness of the bridge system are mostly within the elastic range of line; (2) when the bridge system exhibits an inelastic reaction, the initial effective stiffness is linearized first, and then the the equal energy principle or the equal displacement principle are used to correct the bridge structure; (3) analyze the replacement structure.

The basic principle of the response spectrum method is that the actual seismic wave acting on the structure is composed of complex waves containing a certain 
excellent frequency, and when the excellent frequency of the earthquake coincides with the natural frequency of the structure, the dynamic response of the structure becomes larger.

For the vibration system of free degree, the vibration equation is:

$$
m \ddot{u}(t)+c \dot{u}(t)+k u(t)=-m \ddot{u}_{g}(t)
$$

According to Formula (1), several constants of the natural vibration characteristics of single free degree structure are obtained from the knowledge of structural dynamics.

Undamped natural period (or natural vibration period):

$$
T=2 \pi \sqrt{m / k}
$$

Undamped natural circular frequency (or natural vibration circular frequency):

$$
\omega=2 \pi / T
$$

In the formula, the critical damping ratio (referred to as the damping ratio):

$$
\xi=c / c_{r}=c / 2 \sqrt{k m}=c / 2 m \omega
$$

Critical damping coefficient is $c_{r}=\sqrt{2 \mathrm{~km}}$

Undamped circular frequency is $\omega=\sqrt{\mathrm{k} / \mathrm{m}}$

After the differential solution, the general displacement solution is:

$$
\begin{gathered}
u(t)=e^{-\xi \omega t}\left(u_{0} \cos \omega_{d} t+\frac{\dot{u}_{0}+u_{0} \xi \omega}{\omega_{d}} \sin \omega_{d} t\right) \\
-\frac{1}{\omega_{d}} \int_{0}^{t} \ddot{u}_{g}(t) e^{-\xi \omega(t-\tau)} \sin \omega_{d}(t-\tau) d \tau
\end{gathered}
$$

Due to the existence of damping, the free vibration of the system $u_{1}(t)$ decays quickly to zero, which is generally not considered. Therefore,

$$
u(t)=-\frac{1}{\omega_{d}} \int_{0}^{t} \ddot{u_{g}}(t) e^{-\xi \omega(t-\tau)} \sin \omega_{d}(t-\tau) d \tau
$$

It is the relative displacement response of a single free degree elastic system under the action of earthquake.

Therefore, the absolute acceleration response and the total acceleration response $\ddot{u}(t)+\ddot{u_{g}}(\tau)$ are

$$
\begin{aligned}
& \ddot{u}(t)+\ddot{u_{g}}(\tau)=\frac{\omega^{2}}{\omega_{d}} \int_{0}^{t} \ddot{u_{g}}(\tau) e^{-\xi \omega(t-\tau)} \sin \omega_{d}(t-\tau) d \tau \\
& -2 \xi \omega \int_{0}^{t} \ddot{u_{g}}(\tau) e^{-\xi \omega(t-\tau)}\left[\left(\xi / \sqrt{1-\xi^{2}}\right) \sin \omega_{d}(t-\tau)\right. \\
& \left.-\cos \omega_{d}(t-\tau)\right] d \tau
\end{aligned}
$$

Considering that the damping ratio $\xi$ is small, $\omega_{d}=\omega \sqrt{1-\xi^{2}} \approx \omega$, the formula can be regained. $\ddot{u}(t)+\ddot{u}_{g}(\tau)=\omega \int_{0}^{t} \ddot{u}_{g}(\tau) e^{-\xi \omega(t-\tau)} \sin \omega(t-\tau) d \tau$

It can be seen from Formula (5) that when the acceleration $\ddot{u}_{g}(t)$ of the ground motion during the earthquake is known, as different elastic systems of single free degree have different dynamic characteristics, in seismic design, the designer is most concerned with the maximum response, the maximum along the time history.

The maximum seismic inertial force of the structure

$S_{a}=\left|\ddot{u}(t)+\ddot{u_{g}}(\tau)\right|=\omega\left|\int_{\max }^{t} \ddot{u}_{g}(\tau) e^{-\xi \omega(t-\tau)} \sin \omega(t-\tau) d \tau\right|$

during the duration of the earthquake is:

$$
\begin{gathered}
F=|F(t)|_{\max }=m\left|\ddot{u}(t)+\ddot{u_{\mathrm{g}}}(t)\right|_{\max }=m S_{a} \\
=\frac{\mid \cdot S_{a}}{\left|\ddot{u_{g}}(t)\right|_{\max }} \frac{\mid}{g} m g=\beta k_{h} G=\alpha G
\end{gathered}
$$

In the formula, $\beta=S_{a} /\left|\ddot{u}_{g}\right|_{\max }$ is the power amplification factor, representing the ratio of the maximum absolute acceleration response $S_{a}$ of the structure to the peak acceleration $\left|\ddot{u}_{g}\right|_{\max }$ of the ground motion; $k_{h}=\left|\ddot{u}_{g}\right|_{\max } / g$ is called the horizontal seismic coefficient, representing the ratio of the horizontal ground motion peak acceleration to the gravity acceleration; $\alpha=k_{h} \beta$ is called earthquake influence coefficient.

Complex bridge structure is a structural system with infinite degrees of freedom, and single-matrix response spectrum analysis cannot meet the requirements any more. Therefore, the vibration mode decomposition is required, and the whole structure can be regarded as the elastic system with $\mathrm{N}$ degrees of freedom moving horizontally

on the ground $\ddot{u}_{g}(t)$ (assuming that the seismic wave is a vertical incident shear wave), and the equilibrium equation acting on the $i$ material point is:

$$
m_{i}\left(u_{i}+\ddot{u}_{g}\right)+\sum_{j=1}^{N} c_{i j} \dot{u}_{i}+\sum_{j=1}^{N} k_{i j} u_{i}=0
$$

In the formula, $k_{i j}$ represents the elastic restoring force applied at the point $i$ when the point $i$ produces unit displacement along the direction of motion (the displacement of point $i$ remains at zero); ${ }^{c_{i j}}$ represents the elastic damping force applied at the point $i$ when the point $i$ produces unit velocity along the direction of motion (the velocity of point $i$ remains at zero). 
The matrix vector form of the dynamic equilibrium equation is:

$$
\begin{gathered}
{[m]\{\ddot{u}(t)\}+[c]\{\dot{u}(t)\}+[k]\{u(t)\}} \\
=-[m]\{J\} \ddot{u}_{g}(t)
\end{gathered}
$$

In the formula, $[m],[c]$ and $[k]$ are respectively $\mathrm{N} \times \mathrm{N}$ mass matrix, $\mathrm{N} \times \mathrm{N}$ damping matrix, and $\mathrm{N} \times \mathrm{N}$ stiffness matrix. $\{J\}$ is $\mathrm{N} \times 1$ column vector with the element of 1 , which is called the identification vector and used to identify the application direction of the ground motion.

Assume that all nodes of the structure move in phase with uniform acceleration. The above equation is solved by the mode superposition method:

$$
\ddot{q}_{j}(t)+2 \xi_{j} \omega_{j} \dot{q}_{j}(t)+\omega_{j}^{2} q_{j}(t)=-\gamma_{j} \ddot{u}_{g}(t)
$$

In the formula, $q_{j}(t)$ - the function of time, called the generalized coordinates of the No. $j$ vibration type;

$\xi_{j}$ _ natural vibration damping of No. $j$ vibration type;

$\omega_{j}$ _ natural vibration circle frequency of No. $j$ vibration type;

$\gamma_{j}$ _ participation coefficient of No. $j$ vibration type.

Therefore, the maximum horizontal seismic inertial force of the No. $j$ vibration type and No. $i$ mass point on the structure is:

$$
\begin{aligned}
F_{i j} & =\left|F_{i j}(t)\right|_{\max }=m_{i} \gamma_{j} \phi_{i j}\left|\ddot{\Delta}_{j}(t)+\ddot{u}_{g}(t)\right|_{\max } \\
& =\phi_{i j} \gamma_{j} k_{h} \beta_{j} G_{i}=\phi_{i j} \gamma_{j} \alpha_{j} G_{i}
\end{aligned}
$$

In the formula, $m_{i}$ - mass of No. $j$ vibration type; $\phi_{i j}$ _ the displacement of the mass point $i$ at No. $j$ vibration type;

$\beta_{j}$ _ the dynamic magnification factor of No. $j$ vibration type, $\quad \beta_{j}=\frac{\left|\ddot{\Delta}_{j}(t)+\ddot{u}_{g}(t)\right|_{\max }}{\left|\ddot{u}_{g}(t)\right|_{\max }}$;

$$
\begin{aligned}
& k_{h} \text { _ seismic coefficient, } \\
& \alpha_{j} \text { _ the earthquake impact coefficient of No. } j
\end{aligned}
$$
vibration type, $\alpha_{j}=k_{h} \beta_{j}$;

$G_{i}$ _ the gravity of mass point $i$;

Due to the influence of many random factors, the acceleration response spectrum has great randomness, and the maximum response of each vibration mode does not occur at the same time. In order to obtain seismic resistance simulation results more accurately in engineering practice, the maximum response of each mode is usually calculated by the vibration mode combination method, such as SRSS method and CQC method.

\subsection{Time history analysis}

Time history analysis is a dynamic analysis method that solves the dynamic differential equations of structures iteratively, which is also called elasto-plastic dynamic analysis method[2]. From the time history analysis method, the changing displacement, velocity and acceleration dynamic responses of each mass point with time can be obtained firstly, and then the time history of the internal force and deformation of the component can be calculated. The specific implementation and calculation process of time history analysis method is shown as follows. Firstly, the ground motion time $t$ is divided into a series of time intervals $\Delta^{t}$ according to fixed time interval and quantity, then the whole structural system is calculated as a linear system in each time interval $\Delta^{t}$, and finally the reaction at each moment is gradually determined.

According to the D'Alembert principle, the equation of motion balance of the structural system with multiple degrees of freedom is:

$$
\begin{gathered}
\{U(t)\}=\{\ddot{u}(t)\}+\{J\} \ddot{u}_{g}(t) \\
-[m]\{\ddot{U}(t)\}-\left\{F_{S}(t)\right\}-\left\{F_{D}(t)\right\}=\{0\}
\end{gathered}
$$

In the formula, $\{U(t)\}$ — the absolute displacement and rotation angle of all nodes at the moment $t$;

$[m]$

$\{J\}$

— the identification vector;

$\{\ddot{U}(t)\}$ the moment $t$;

$\left\{F_{S}(t)\right\}$ the absolute acceleration of all nodes at

the restoring force caused by the deformation of the system that enables the mass point to restore from the vibration position to the equilibrium position;

$\left\{F_{D}(t)\right\}$ the damping force that gradually attenuates structural vibration.

The restoring force and damping force can be respectively written as follows:

$$
\begin{aligned}
& \left\{F_{S}(t)\right\}=[k]\{u(t)\} \\
& \left\{F_{D}(t)\right\}=[c]\{\ddot{u}(t)\}
\end{aligned}
$$

In the formula, $[k]$ _ stiffness matrix of structural system;

$[c]$ _ damping matrix of structural system; 
By substituting Formula (11), (13), and (14) into Formula (12), the equation of motion can be obtained:

$$
\begin{aligned}
& -[m]\{\ddot{u}(t)\}+[c]\{\dot{u}(t)\}+[k]\{u(t)\} \\
& =-[m]\{J\} \ddot{u}_{g}(t)
\end{aligned}
$$

In the calculation process, the acceleration horizontal component and the time curve recorded during the earthquake are divided into small time periods $\Delta t$, and then direct integration is performed by the vibration equation (15) for each time period, thereby obtaining the displacement, velocity and acceleration of structural system at each moment and then to calculate the internal force of the structure.

\section{Comparative analysis of response spectrum method and time history analysis method}

\subsection{Comparison of principle content}

The essence of the calculation principle of the response spectrum method is the vibration mode decomposition algorithm, while the essence of the time history analysis method is the integral algorithm. Both the response spectrum method and the time history analysis method can reflect the seismic response characteristics of the structure under the action of earthquake. Li Zhengying[3] and others assumed that the same large-span bridge structure is under the same earthquake action, that is, when the seismic waves are the same, it is found that the seismic response calculated by the response spectrum method and the time history analysis method has a certain degree of comparability.

\subsection{Advantages and disadvantages of response spectrum method}

The response spectrum method is to statically solve the structural dynamic problem, the calculation process is simple, the calculation amount is small, and the maximum value of the structural seismic response can be effectively calculated, so it has been widely used in practice.

The response spectrum method assumes that the structure is an elastic state, and the elastic response spectrum theory is used for the analysis of the seismic force of the structure. Therefore, the response spectrum method is essentially only applicable to elastic bridge structures, to conduct linear analysis on it. Ding Jianguo[4] and others pointed out that the response spectrum analysis method is based on the maximum seismic response of multiple seismic waves, and the final result is more conservative.

In reality, earthquake is a random load. In a strong earthquake, long-span bridge structure will enter a nonlinear state in most cases. Therefore, Liu Chunguang[5] and others believe that the response spectrum method cannot reflect the influence of ground motion duration and nonlinearity on the structure. At the same time, Lin Jiahao believes that when the seismic response analysis on bridge structure system of multiple degrees of freedom is carried out by using the response spectrum method, the multi-vibration type decomposition method has some problems of vibration mode combination.

\subsection{Advantages and disadvantages of time history analysis}

The time history analysis method is a method for solving the structural dynamic equilibrium equation by the integral method, so the nonlinear analysis of the structure can be performed. The method is based on a number of actual seismic waves and is a more accurate structural dynamic analysis method that can simulate the seismic response of the structure over the time period of the entire seismic duration, and then obtain the dynamic response characteristics, locations where structures are damaged and structural failure modes of the structure during the earthquake.

When using the time history analysis method to analyze large-span bridges, the dynamic equilibrium equation established at the same time is complicated because of the large number of basic units, so the solution speed and convergence time are slow. Meanwhile, since the calculation analysis time step is usually set to below $0.001 \mathrm{~s}$, and the total calculation time is set to be more than $10 \mathrm{~s}$, the computer is time consuming and the resulting file is large.

Although the time history analysis method can obtain numerically accurate analysis results, there are some parameters that are difficult to determine, such as the parameter input of ground motion, nonlinear dynamic characteristics of structural members, structure-foundation-soil interactions and so on. $\mathrm{Li}$ Xiaojun[6] studied the impact of pile-soil effect and ground motion parameters on the calculation results of time history analysis method. Biao Fangjun[7] studied the influence of nonlinear dynamic characteristics of structural members on the seismic response of bridges. They found that the difference in parameter settings will have a certain regular effect on the calculation results.

\subsection{The application scope of response spectrum method and time history analysis method}

As a simple and practical seismic resistance analysis method, the response spectrum analysis method is widely used in seismic resistance analysis of medium and small-span bridges at present. For large-span bridges, due to their large nonlinear structural response in earthquakes, the response spectrum method is rarely used in the accurate seismic resistance analysis of large-span bridge structures.

Both the response spectrum method and the elastic time history method can be used for the response analysis of continuous beam bridges experiencing multiple longitudinal earthquakes or multiple transverse earthquakes. Under the rare earthquakes, some structural members have entered the plastic stage, and applying 
elastic model will seriously amplify the bending moment at the bottom of the pier. Therefore, using the elastoplastic time history method to conduct seismic response analysis on the bridge structure can obtain more accurate structural dynamic response characteristics.

\section{Conclusion and outlook}

At present, the most commonly used methods for seismic resistance design of bridge structures are response spectrum method and time history analysis method. This paper introduces the calculation principles of these two methods respectively, and summarizes the advantages and disadvantages and the application scope of each method, to provide a reference for researchers to design the seismic resistance of bridge structures.

(1) The reaction spectrum method is simple to calculate, and can effectively calculate the maximum value of structural seismic response, which is widely used in practice. However, this method can only conduct linear analysis on the structure, but cannot reflect the influence of ground motion duration and nonlinearity on the structure. This method is suitable for the response analysis of medium and small-span bridges experiencing multiple longitudinal earthquakes or multiple transverse earthquakes.

(2) Compared with response spectrum method, the time history analysis method is a more accurate structural dynamic analysis method that can perform nonlinear analysis on the structure. But the computational analysis of the time history analysis method takes longer time, and the parameter setting of the ground motion parameter input, the nonlinear characteristics of the component and the interaction between the structure and the foundation would have a great influence on the simulation analysis results.

(3) The response spectrum method is only applicable to the response analysis of medium and small-span bridges in the cases of multiple longitudinal earthquakes and multiple earthquakes. The time history analysis method is also applicable to the response analysis of medium and small-span bridges under rare earthquakes and seismic resistance analysis of large-span bridges.
Many scholars at home and abroad have carried out a lot of research work on response spectrum method and time history analysis method. On the basis of this, methods have been improved and revised, and some achievements have been made. The calculation of the response spectrum method is simple, and meets the requirements of national design specifications. The research on the response spectrum method applicable to multi-point activation has broad application prospects. The time history analysis method relies a lot on ground motion input, and the ground motion power function in random vibration analysis needs further study.

\section{References}

1. Ye Aijun, Guan Zhongguo. Seismic Resistance of Bridge (Second Edition) [M]. Beijing: China Communications Press, 2011.

2. Ernesto H Z, Vanmarcke E H. Seismic Random Vibration Analysis of Multi-support Structural Systems [J]. Journal of Engineering Mechanics, ASCE, 1994, 120: 1107-1128.

3. Li Zhengying. Seismic Response Analysis of Long-Span Arch Bridges under Spatial Ground Motion [J]. Journal of Chongqing University: Natural Science Edition, 2009(8), 24-29.

4. Ding Jianguo. Elastoplastic Response Spectrum and Its Application in Seismic Resistance Design [J]. Journal of Nanjing University of Science and Technology (Natural Science), 2007(6):780-783.

5. Liu Chunguang, Lin Gao. Study on Seismic Resistance Performance of Push-over Method of Bridge Structure [J]. Journal of Dalian University of Technology, 2005, 5 45(3): 395-400.

6. Li Xiaojun. Step-by-step Integration Method for Solving Dynamic Equations in Earthquake Engineering [J]. Engineering Mechanics, 2007(2): 67-68.

7. Biao Fangjun, Yan Xiaoming. Dynamic Elastoplastic Time-History Analysis and Its Application [J]. Civil Engineering and Architecture, Shenzhen, 2006, 3 (1): 26-29. 